\title{
Prominent Cutaneous Manifestation of COVID-19: A Case Report
}

\author{
Authors: \\ Cristina Galván-Casas, ${ }^{1}$ Juan F. Ochoa-Bermúdez, ${ }^{2}$ Sara Muñoz- \\ Ordoñez, ${ }^{2}$ Álvaro Tovar-Cock, ${ }^{3}$ Telma Meizoso ${ }^{4}$ \\ 1. Dermatology Department, Hospital Universitario de Móstoles, Madrid, Spain \\ 2. Universidad del Rosario, Bogotá, Colombia \\ 3. Medical Department, Hospital Universitario Centro Dermatológico Federico Lleras \\ Acosta, Bogotá, Colombia \\ 4. Pathology Department, Hospital Universitario de Móstoles, Madrid, Spain \\ *Correspondence to juanfelipeochoab@gmail.com
}

Disclosure: $\quad$ The authors have declared no conflicts of interest.

Received: $\quad 12.10 .20$

Accepted: $\quad 08.03 .21$

Keywords: Coronavirus disease (COVID-19), cutaneous manifestations, skin.

Citation: $\quad$ EMJ. 2021; DOI/10.33590/emj/20-00247

\section{Abstract}

Extrapulmonary signs of coronavirus disease are becoming an important tool for patient diagnosis; this is particularly true for skin manifestations as they are visible to both the patient and physician. In this case report, the authors describe a case in which cutaneous manifestations were the most noticeable and prominent symptom in a patient with coronavirus disease.

\section{INTRODUCTION}

The new severe acute respiratory syndrome coronavirus 2 (SARS-CoV-2) pathogen and the subsequent coronavirus disease (COVID-19) mainly affect the lung. ${ }^{1}$ Respiratory symptoms are key for diagnosis, however, increasing knowledge has led to the understanding that COVID-19 induces multiorgan pathology. The first descriptions of clinical symptoms reported that $0.2 \%$ of patients admitted to hospitals in China presented with a skin rash. ${ }^{1}$ Following this, in Italy $20.4 \%$ of 88 admitted patients treated by dermatologists presented with symptoms of the skin. ${ }^{2}$

In April 2020, the first comprehensive classification of skin manifestations in patients within the full spectrum of COVID-19 severity, from intensive care, hospital wards, and home

care, as well as those without symptoms, was published. ${ }^{3}$ The study analysed 375 patients with PCR-confirmed diagnosis or with suspected diagnosis, meeting the European Centre for Disease Prevention and Control (ECDC) clinical criteria. Although following certain patterns, skin manifestations showed extreme variability. Five skin patterns were defined: pseudo-chilblain, vesicular, urticarial, maculopapular, and livedo/ necrosis. Each pattern usually associated with a different age category, evolutionary moment of the process, and systemic severity. ${ }^{3}$ The majority of these manifestations were non-specific and their cause-effect relationship with the virus is not fully established. The limited access to confirmatory tests and concurrence of different drugs to treat the disease make it difficult to reach any conclusions. The authors present the case of a patient in whom the most striking COVID-19 manifestation was dermatological. 


\section{CASE REPORT}

A 47-year-old female, with no relevant pathological or family medical history, presented with a dry cough, diarrhoea, and chills. Three days later, she developed a headache, myalgia, and a fever $\left(39^{\circ} \mathrm{C}\right)$. On Day 7 , she observed anosmia and improvement of the previous symptoms. On Day 16, coinciding with worsening of headache, myalgia, chills, fever, and anosmia, she presented with a skin rash, ageusia, and nausea without vomiting. The skin eruption consisted of itchy and painful lesions, first on the abdomen and later affecting the back and limbs, including the dorsum of the hands. On examination, multiple non-scaling erythematous papules and plaques were noted.

The patient was attended to in the emergency unit. Her vital signs were a temperature of 36.4 ${ }^{\circ} \mathrm{C}$, blood pressure of $130 / 80 \mathrm{mmHg}$, heart rate of 89 beats per minute, and oxygen saturation of $98 \%$. Her chest X-ray was reported as normal. She was discharged home and prescribed the treatment of azithromycin 500 mg every 24 hours and dexchlorpheniramine $6 \mathrm{mg}$ every 8 hours for 5 days.

In the days following, the lesions became larger and oedematous with pseudo-vesicular appearance on the dorsum of the hands (Figure 1). On Day 21, she was evaluated using teledermatology. Face-to-face consultation was recommended for examination and COVID-19 testing. On examination, her temperature was $35.5{ }^{\circ} \mathrm{C}$, oxygen saturation was $100 \%$, chest X-ray was normal, and nasopharyngeal PCR for SARS-CoV-2 was negative. Histopathological examination showed hyperkeratosis, parakeratosis, and acanthosis. In the dermis, a dense, sleeve-like perivascular lymphohistiocytic infiltrate with abundant eosinophils pervaded throughout the dermis. Abundant eosinophils were perivascular and interstitially located. Capillaries were conspicuously dilated and engorged with red blood cells but no fibrinoid necrosis was identified (Figure 2A). Deep eccrine glands were surrounded by moderate lymphoid and eosinophil infiltration (Figure 2A). These histopathological features have previously been observed in the skin lesions of patients with COVID-19 and have been defined as having a 'mini-chilblain' pattern (Figure 2A and 2B). ${ }^{4}$
The patient had follow-up home visits and was treated with paracetamol, dexchlorpheniramine, and topical mometasone once per day on the lesions. In the following days, the symptoms persisted and the lesions developed a central crusty dark area.

On Day 28, the itching began to improve, and the papules flattened and turned into hyperpigmented macules concentrically surrounded by an ecchymotic halo, sparing the skin immediately adjacent to the macules. The resolution of the lesions and the itching was gradual, totally disappearing 2 months later. Three months after the onset of symptoms, the antibody test for SARS-CoV-2 was positive. Four months later, the patient presented massive shedding. The hair loss was diffuse throughout the scalp, typical of telogen effluvium; however, they also presented round bald patches with an active border, typical of alopecia areata.

\section{DISCUSSION}

COVID-19-associated skin manifestation reports are numerous; however, the authors believe that they are under-reported. There are several possible causes of this: the difficult recognition in patients with mild or absent general symptoms; the lack of knowledge regarding the manifestations of COVID-19; the difficulty accessing diagnostic tests; the clinical similarities among other dermatological diseases; and the self-resolving nature or short-term evolution. Due to the strain COVID-19 had on the healthcare system, skin examinations were not a priority among hospitalised or symptomatic patients, particularly in the most critical cases. Additionally, admission areas with restricted access made patient examination even more difficult, often preventing photographic record and obtainment of informed consent.

In COVID-19 pathogenesis, the host's response plays an important role. The first line of defence against SARS-CoV-2 is the innate immune system, specifically interferon-I production and natural killer (NK) cell intervention. ${ }^{5}$ The virus dampens the initial interferon-I response to achieve uncontrolled viral replication. Subsequently, humoral and cell-mediated adaptive immunity are activated. 


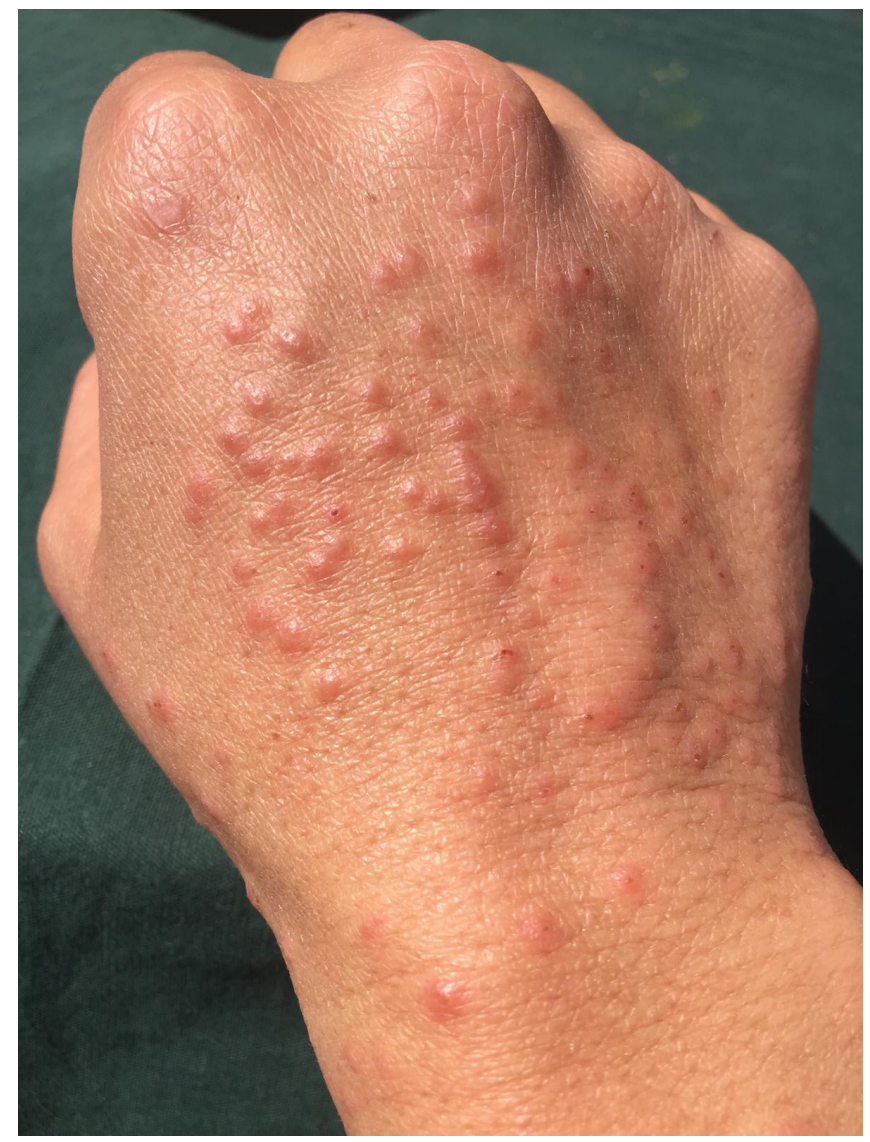

Figure 1: Large oedematous papules on the dorsum of the hand.
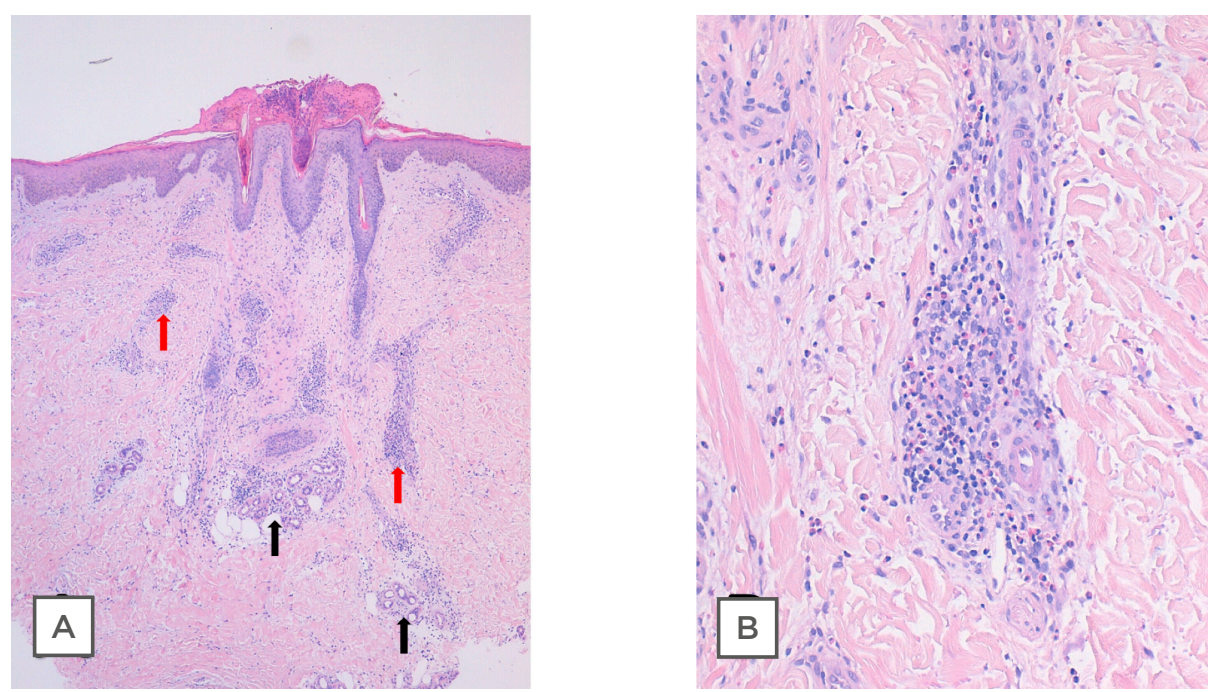

\section{Figure 2: Histological plates stained with haematoxylin and eosin.}

A) 4x: Panoramic skin biopsy showing hyperkeratosis, parakeratosis, and acanthosis. In the dermis is a dense, sleevelike perivascular lymphohistiocytic infiltrate with abundant eosinophils. Eosinophils were abundant, perivascular, and interstitially located. Capillaries were conspicuously dilated and engorged with red blood cells, but no fibrinoid necrosis was identified (red arrows). Deep eccrine glands were surrounded by moderate lymphoid and eosinophil infiltration (black arrows). B) 20x: Photograph at high magnification showing lymphohistiocytic infiltrate with the presence of eosinophils. 
Inflammatory cytokines and antibodies promote the recruitment of neutrophils, monocytes, and macrophages, stimulating further development of additional inflammatory cytokines. In critical cases, activation of the coagulation pathway occurs with consumption of coagulation factors, ${ }^{6}$ leading to multiorgan thrombotic manifestations, including thrombosis of pulmonary and skin vessels. ${ }^{7}$ In the majority of infected patients, the immune response leads to viral clearance. However, if the effector response results in an antibody-dependent enhancement (ADE), which favours the entry of the virus into macrophages, it will result in the increase of proinflammatory cytokines such as TNF and IL6. ${ }^{5}$ This phenomenon, known as cytokine storm, is the cause of tissue damage and multiorgan dysfunction and could also manifest on the skin. ${ }^{8}$ Nevertheless, as well as in other organs, the different manifestations of the disease in the skin must be related to the diverse immune responses of the host. ${ }^{9}$

In addition to the pattern classification described prior, another interesting study proposed a classification with two groups: exanthematic and inflammatory lesions; and vasculitic and vasculopathic lesions. In the second group, the authors differentiated the purpuric vasculitic pattern, which has a low incidence, from the petechial component in some exanthematic lesions. ${ }^{10}$ In those patterns cited, different histological and immunohistochemical findings and distinct pathophysiological hypotheses related to different host immune responses were described. A robust innate immunity response would be related to chilblain-like lesions, whereas the hypercoagulable state and cytokine storm would induce pauci-inflammatory thrombotic microangiopathy with the development of livedo or necrotic lesions. ${ }^{7}$ Direct viral cellular damage of angiotensin-converting enzyme 2 (ACE2) receptors on basal cells would induce the vesicular pattern. Antigen-antibody complex deposits, non-specific activation of mast cells, viral endothelial damage (rich in ACE2), complement activation, and activation of the bradykininkallikrein system have been postulated as cause of the urticarial pattern. ${ }^{11,12}$ In many occasions, other intercurrent causes, such as the reactivation of viral diseases or toxicoderma, cannot be ruled out.
The interest in the reported case lies in the clinical presentation of the greatly expressed skin manifestations, which is not previously described. The skin lesions were complex, longlasting, and highly symptomatic, representing the most striking symptom of the patient's symptomatology. These lesions concurred with mild general symptoms in the second week after onset and lasted for 2 months. They produced intense itching with poor response to antihistamine treatment. They evolved from small papules without scaly component to larger, oedematous, pseudo-vesicular papules resembling erythema elevatum diutinum. Later, the papules developed a central scaling with target-like images reminiscent of erythema multiforme. In the time they were flattening into macules, they presented striking concentric ecchymotic haloes. Four weeks after the disappearance of the skin lesions, intense telogen effluvium and alopecia areata appeared: two types of hair loss with different physiopathology, which do not usually appear concomitantly. No manifestations were found in mucous membranes or nails.

Taking into account the main patterns described, a maculopapular rash can be identified. Its onset coincided with the rest of the systemic symptoms, although these had already been present for 2 weeks. The effects of the medication should not be considered since the onset of the lesions was prior to the administration of any treatment. The resolution phase, with ecchymosis surrounding the lesions, cannot be explained by scratching since it had a rounded and concentric distribution around the papules and there were no linear erosions. The relationship of this sign with endothelial damage and the tendency towards hypercoagulability in patients with COVID-19 is not supported by the histological study of the biopsy. Appearance of telogen effluvium after infectious diseases is well described and the authors unreported experience supports that it is not uncommon after COVID-19.13 Alopecia areata is also already described..$^{14}$ Traditionally, it has been related to immune disorders and stress; the patient reported an intense emotional burden due to the pandemic, the isolation situation, and the discomfort caused by the skin rash. Interestingly, both types of alopecia have been diagnosed more frequently after the peaks of the pandemic compared to previous periods. ${ }^{14,15}$ 
Based on the literature reviewed, the authors assume that this patient's specific and dysregulated immune response led to her diverse and prolonged dermatological manifestations. Reporting cases is vital to expand information and provide a more precise approach to COVID-19 skin manifestations. The skin is an organ accessible to visual examination and can be extremely helpful in regard to this worldwide relevant disease.

Note: the patient consented to the presentation of the case and the images. This case is one of 375 cases in a published cohort study, ${ }^{3}$ and this case report has approval from the publishing journal.

\section{References}

1. Guan W et al. Clinical characteristics of coronavirus disease 2019 in China. N Engl J Med. 2020;382(18):1708-20.

2. Recalcati S. Cutaneous manifestations in COVID-19: a first perspective. J Eur Acad Dermatology Venereol. 2020;34(5):e212-3.

3. Galván Casas C et al. Classification of the cutaneous manifestations of COVID-19: a rapid prospective nationwide consensus study in Spain with 375 cases. Br J Dermatol. 2020;183(1):71-7.

4. Gianotti R et al. Similarities in cutaneous histopathological patterns between COVID-19-positive and COVID-19 high-risk patients with skin dermatosis. Acta Derm Venereol. 2020;100(15):1-5.

5. Wang SF et al. Antibody-dependent SARS coronavirus infection is mediated by antibodies against spike proteins. Biochem Biophys Res Commun. 2014;451(2):208-14.
6. Tang $\mathrm{N}$ et al. Abnormal coagulation parameters are associated with poor prognosis in patients with novel coronavirus pneumonia. J Thromb Haemost. 2020;18(4):844-7.

7. Magro $\mathrm{C}$ et al. Complement associated microvascular injury and thrombosis in the pathogenesis of severe COVID-19 infection: a report of five cases. Transl Res. 2020;220:1-13.

8. Lee $\mathrm{N}$ et al. Anti-SARS-CoV lgG response in relation to disease severity of severe acute respiratory syndrome. J Clin Virol. 2006;35(2):179-84.

9. Criado PR et al. Lessons from dermatology about inflammatory responses in Covid-19. Rev Med Virol. 2020;30(5):e2130.

10. Marzano AV et al. Cutaneous manifestations in patients with COVID-19: a preliminary review of an emerging issue. $\mathrm{Br} \mathrm{J}$ Dermatol. 2020;183(3):431-42.
11. Criado PR et al. What the physicians should know about mast cells, dendritic cells, urticaria, and omalizumab during COVID-19 or asymptomatic infections due to SARS-CoV-2? Dermatol Ther. 2020;33(6):e14068.

12. Marzano AV et al. Varicella-like exanthem as a specific COVID-19associated skin manifestation: multicenter case series of 22 patients. J Am Acad Dermatol. 2020;83(1):2805.

13. Chu CB, Yang CC. Dengue-associated telogen effluvium: a report of 14 patients. Dermatologica Sin. 2017:35(3):124-6.

14. Sgubbi $P$ et al. Alopecia areata in a patient with SARS-Cov-2 infection. Dermatol Ther. 2020;33(6):e14295.

15. Kutlu Ö, Metin A. Relative changes in the pattern of diseases presenting in derma-tology outpatient clinic in the era of the COVID-19 pandemic. Dermatol Ther. 2020;33(6):e14096. 